\title{
A Sepse como Estado Disfuncional do Hospedeiro: Novas Definições e Paradigmas Diagnósticos
}

Raphaell Pinto de Arruda Trindade ${ }^{1}$, Edson Marques da Silva Filho ${ }^{1}$ Elves Anderson Pires Maciel

Palavras-chave: sepse, choque séptico, síndrome da resposta inflamatória sistêmica Key words: sepsis, septic shock, SRIS

INTRODUÇão

A sepse, como condição clínica, vem sendo estudada desde os povos antigos, tendo sua terminologia derivada do grego "sepo", que significa putrefação. Porém, relatos de sua descrição remotam mais de 2.500
anos na China. Anos depois, Roger Bone introduziu o conceito moderno para sepse, sendo uma condição que englobava a síndrome de resposta inflamatória sistêmica (SRIS).

Neste contexto, a definição de sepse, entendida como uma síndrome decorrente da interação de um agente infeccioso com a resposta do hospedeiro, vem sendo atualizada consecutivamente nas últimas três décadas, sendo a última publicada em 2016, o objeto dessa revisão.

A sepse representa um importante problema de saúde pública no mundo, com 17 milhões de casos estimados anualmente, com elevada morbimortalidade. No Brasil, os dados disponíveis apontam para uma letalidade maior do que a dos países desenvolvidos, ultrapassando os $50 \%$ nas formas mais graves (choque séptico), além de desfechos piores nos pacientes atendidos pelo Sistema Único de Saúde (SUS). ${ }^{2}$

O melhor entendimento dos fenômenos fisiopatoló gicos que ocorrem quando um agente infeccioso desencadeia uma resposta do hospedeiro, que por si pode ser lesiva, vem aumentando progressivamente, demandando uma contínua atualização do conceito de sepse. Acompanhando essa evolução no conhecimento dos mecanismos, se faz necessária a atualização dos critérios que definem a síndrome sepse, que, idealmente, devem ser altamente acurados, para que possam ser úteis, tanto na prática clínica quanto na pesquisa.

A EVOLUÇÃO DAS DEFINIÇÕES DA SEPSE Antes da decada de 1990, com base no entendimento vigente, que associava sepse à presença de bactérias na corrente sanguinea, termos imprecisos e inespecíficos, tais como septicemia, síndrome séptica, infecção generalizada, eram utilizados comument para designar sepse. Posteriormente, a constatação da participação de várias citocinas produzidas pelo hospedeiro na patogênese e lesão tecidual ocorrid durante a infecção levaram ao conceito de respost inflamatória sistêmica. Concomitantemente, surgiam propostas de terapias que pudessem bloquear ess cascata inflamatória, reduzir os danos ao hospedeiro consequentemente, a letalidade.

Nesse contexto, em 1992, um consenso, chefiado por Roger C. Bone, foi desenvolvido pela SCCM (Society of Critical Care Medicine), ACCP (American College of Chest Physicians) e a ESICM (European Society of Critical Care Medicine), com o intuito de criar critérios objetivos para sepse e síndromes re lacionadas, assim com abandonar termos obsoleto (como septicemia), que pudessem ser usados para seleção de pacientes, para inclusão nos ensaios clínicos de novas drogas, principalmente as anticitocinas (Sepsis-1). ${ }^{3}$

No entanto, desde o início, o Sepsis-1 foi criticado, pela sua sensibilidade exagerada e baixa especificidade, sem capacidade de diferenciar infecções triviais (como um resfriado comum), ou mesmo processos não infecciosos (tais como pancreatite), de infecções graves.

Por isso, os critérios foram ampliados, mantendo mesma base fisiopatológica de uma resposta inflam tória sistêmica, e publicados em 2012 (Sepsis-2). ${ }^{4}$

Assim, a sepse foi definida como uma infeção com SRIS, "sepse grave" como sepse associada a disfunções orgânicas ou hipotensão e "choque séptico" como sepse grave com choque a despeito de ressuscitação volemica. As definiçoes foram amplamente conhecid desde então e durante os primeiros 10 anos mais de 800 estudos e ensaios clinicos, que focavam principalmente as ações terapêuticas, foram publicados, crian- do-se a necessidade de revisão das definições que englobassem ideias que melhor refletiriam a fisiopatogênese da condição clínica, havendo a formulação o segundo consenso publicado em 2012, o Sepsis-2 Abaixo, no quadro 1, estão os critérios de SRIS:

Quadro 1. Os critérios da Síndrome da Resposta Inflamatória Sistêmica

\section{ar}

Critérios de SRIS

Dois ou mais

- Temperatura $>38^{\circ} \mathrm{C}$ ou $<36^{\circ} \mathrm{C}$

Frequência cardíaca $>90 \mathrm{~min}$

min ou $\mathrm{PaCo}_{2}<32 \mathrm{mmH}$

Leucograma $>12.000 / \mathrm{mm}^{3}$ ou $<4.000 / \mathrm{mm}^{3}$

Até este consenso, a proposição de que a sepse correspondia a uma combinação de sinais e sintomas da síndrome da resposta inflamatória sistêmica era deendida pelos especialistas que acreditavam na potencialidade dos critérios de SRIS para um mapeamento epidemiológico e avaliação do sucesso das estratégias e tratamento nos últimos 25 anos.

Em 2016, as mesmas sociedades promoveram nova conferência de consenso, logo com a publicação recente da força-tarefa da SCCM, que promoveu uma revisão sistematica que redefiniu os criterios e o conceito da sepse: $3^{\circ}$ Consenso Internacional para Definição de Sepse e Choque Séptico (Sepsis-3).

Pela primeira vez, inicialmente, procurou-se definições em dados disponiveis na literatura e não na opinião de especialistas. Alem disso, os criterios da Sindrome da Resposta Inflamatória Sistemica (SIRS) passaram a não ser mais requeridos para o diagnóstico de sepse. Com isso, sepse passa a ser definida como uma disfunção orgânica ameaçadora à vida secundária à resposta desregulada do hospedeiro a uma infecção.

A partir desta compreensão, a sepse passa a ser encarada como um estado de maior gravidade e/ou complicação de uma infecção com disfunções orgânicas documentadas. Consequentemente, redundâncias em termos previamente empregados foram abandonadas, como a "síndrome séptica", "septicemia" e "sepse grave". Ao longo do tempo, isso será importante em termos de promover melhor percepção da sepse por profissionais de saúde e leigos, associando-se o nome "sepse" a um quadro grave.
Diferentemente de outras condições ameaçadoras à vida, como o infarto agudo do miocárdio, teste com elevada acurácia específicos e rápidos para o se diagnóstico ainda não são validados. Consequentemente, o desenvolvimento de exames diagnósticos, como marcadores bioquímicos ou métodos de rastreio de pacientes com elevado risco de desenvolvimento de sepse, necessita da compreensão da entidade orgânica, se única ou múltipla, e se apresentam níveis de acometimentos diferentes.

\section{O SEPSIS-3 E O USO DE QSOFA PARA RAS-} TREIO DA SEPS

As definições e o reconhecimento da sepse a partir de sinais comuns descritos nas definições de SRIS, como pirexia e neutrofilia, fazem parte da definição usual de infecções e não garantem, diretamente, o reconhecimento de disfunções orgânicas associadas ao novo conceito da sepse. Neste as pecto, os componentes dos criterios de SRIS podem significar uma resposta do organismo à infecção, que pode ser adaptativa.

Algumas evidências mostram que os critérios de SRIS podem ser muito sensíveis, o que pode prejudicar sua especificidade para sepse, necessitando, portanto, de novos instrumentos e escores que visem um melhor reconhecimento e rastreio da sepse.

A proposição das novas definições ocorrem no reconhecimento das alterações da resposta do hospedeiro à infecção e à necessidade de valorização das disfunções secundárias. Estas, por estarem ocultas, devem ter sua presença considerada e reconhecida mais facilmente. Infecções ocultas e mal identificadas perpetuam disfunções que tendem a se manter ocultas.

Definiu-se, portanto, sepse como disfunção orgânica para preenchimento e $O$ aumento em 2 pontos escore SOFA (Sequential Organ Failure Assessment) como consequência da infecção (tabela 01). A parti de uma análise multivariada de dados, foram identificadas 3 variáveis clinicas que mantinham o mesmo valor preditivo positivo, em predizer mortalidade, se comparado aos seis elementos oniginais do SOFA em ambiente não-crítico. Com isto, foi descrito o quick SOFA ou qSOFA e seus elementos. Alteração do estado de consciência: escala de coma de Glasgow de 13 ou menos, pressão arterial sistolica menor ou igual a $100 \mathrm{~mm}$ e frequência respiratoria maior ou igual a 22 min. Consequentemente, este instrumento simples fácil de uso foi criado para não fazer parte da definição de sepse, ao contrário do SRIS. ${ }^{6,10}$ 
Tabela 1. SOFA - Sequential Organ Failure Assssment. (extraido de epmjournal.blogspot.com.br)

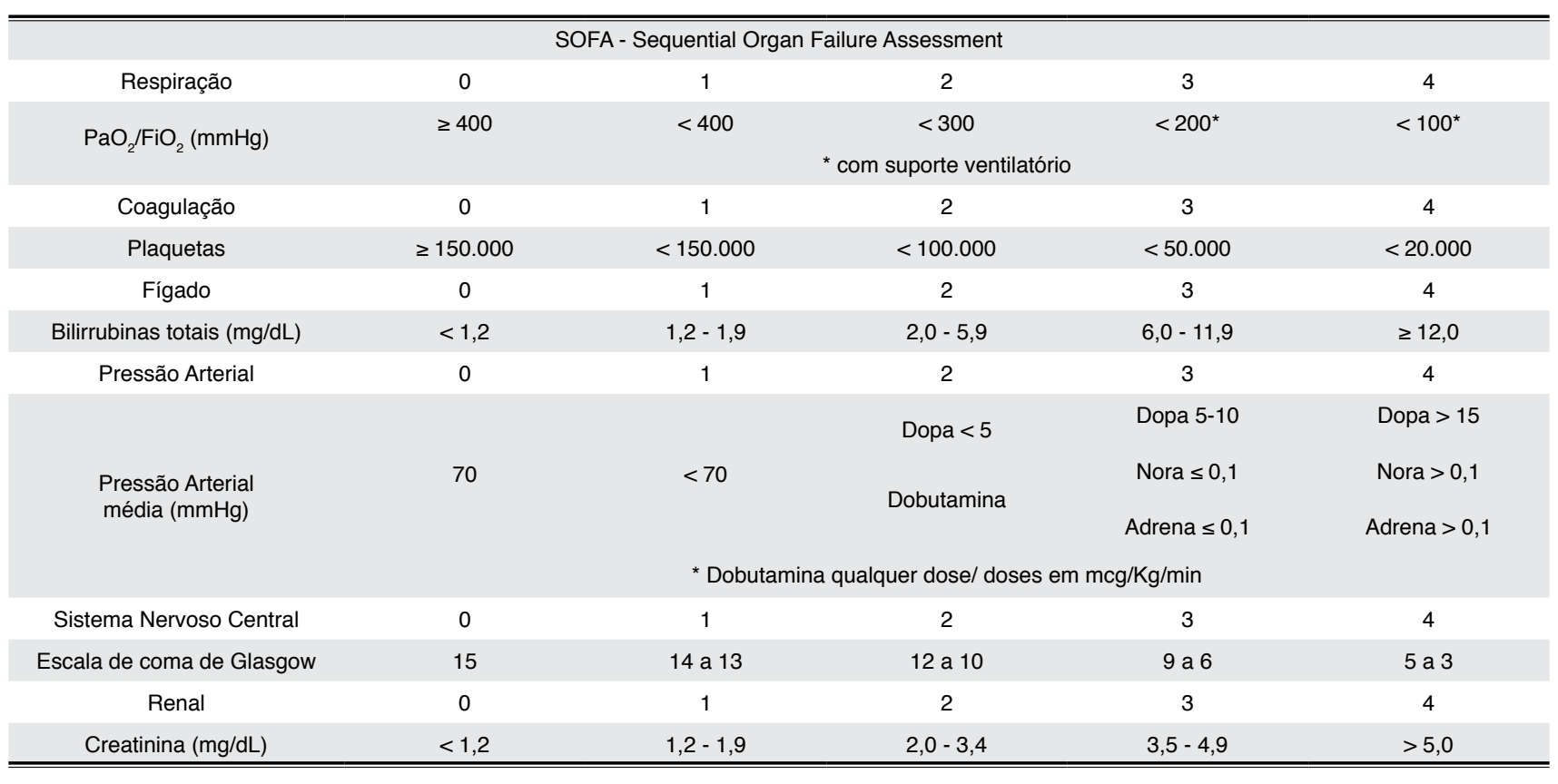

A força-tarefa que promoveu a redefinição dos critérios de sepse (Sepsis-3) recomenda que, a partir da identificação dos pacientes sujeitos ao diagnóstico de sepse, seja aplicado o escore qSOFA e, juntamente com critérios e impressão clinica, como o sítio da infecção, seja feita a identificação da infecção e consequentemente dos pacientes com grande probabilidade de sepse. A infecção presumida, segundo a definição, apresenta um caráter e subjetividade.

Abaixo, na tabela 2, segue o algoritmo sugerido pelo Sepsis-3 para o diagnóstico de sepse.

Tabela 2. Operacionalização dos critérios diagnósticos para identificação dos pacientes com sepse e choque séptico. qSOFA; quick SOFA. PAM; Pressão Arterial Média; ECG; Escala de Coma de Glasgow. (Extraído e traduzido de Third Consensus Definitions for Sepsis and Septic Shock.)

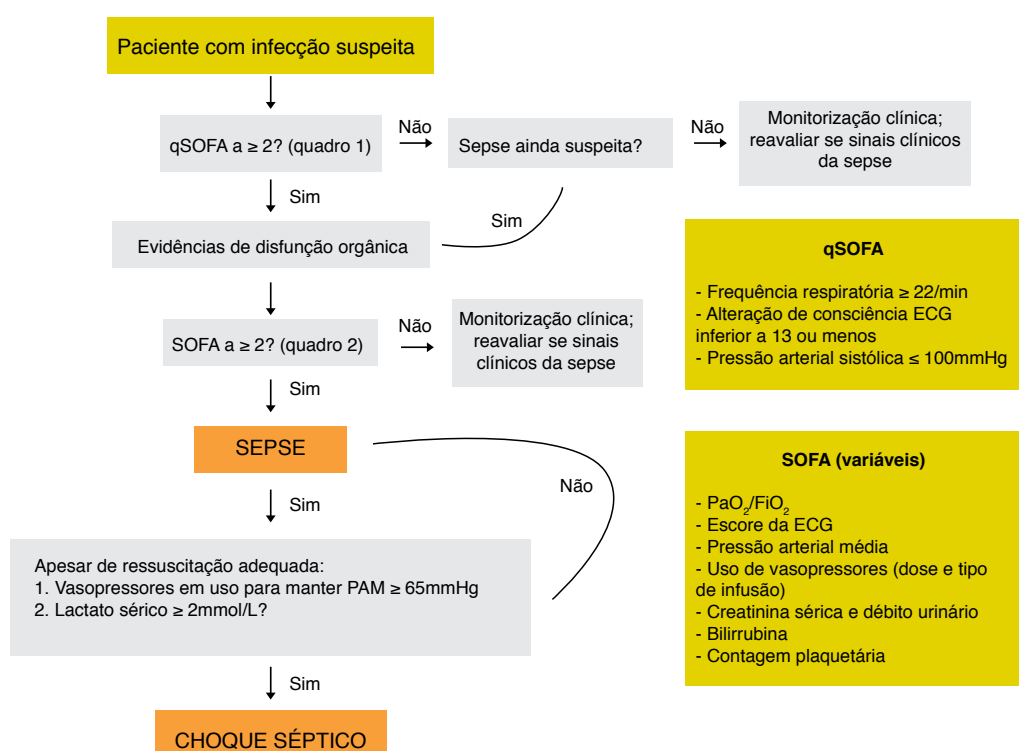

Choque séptico passou a ser definido como preCho de hipotensão com necessidade de vasopressores para manter pressão arterial média $\geq 65 \mathrm{mmHg}$, associada a lactato $\geq 2 \mathrm{mmol} / \mathrm{L}$, após adequada ressuscitação volêmica. Assim, a hiperlactatemia é um componente necessario, se disponivel, para a definição de choque septico, pois consideram-se alteraçoes celulares e metabólicas integrantes do estado de choque. Os novos conceitos limitam os criterios para definir presença de disfunção organnica e selecionam uma população mais gravemente doente. Estes critérios podem, consequentemente, desconsiderar disfunçōes localmente instaladas que, acompanhadas de infecção, estão associadas a um aumento de $10 \%$ de mortalidade intra-hospitalar. Se houve evolução para choque septico, essa mortalidade atinge $40 \% .6,9$

Assim como o SOFA, o qSOFA não pode ser considerado integrante dos criterios de sepse, como os componentes do SRIS, e sim como instrumento útil em varias facilidades hospitalares, como enfermarias ou pronto-atendimento de emergencia, que possam iniciar o julgamento clinico da identificação da sepse promovendo seu tratamento adequado.

O reconhecimento das novas definições e o uso do qSOFA são endossados pelo último consenso e po diretrizes do manejo da sepse e do choque séptico, publicado pela Campanha de Sobrevivencia à Sepse (SSC - Surviving Sepsis Campaign) do ano de $2016 . "$ Apoiados na ideia de apoio à beira leito, o qSOFA pode ser usado para que 0 aumento da suspeita de sepse seja realizado e determine uma ação diretiva. $O$ valor preditivo positivo da ferramenta vem sendo estudado e, recentemente, foi demonstrado que $75 \%$ dos pacientes que apresentavam sepse presumida tinham, pelo menos, dois pontos no qSOFA quando alocados

De forma controversa, o qSOFA é um escore de gravidade, derivado do SOFA, cujo desenvolvimento e validação foram para identicar pacientes con co de óbito ou permanencia na UTI por mais de 3 dias. A ferramenta como estrategia de triagem de pacientes ainda não foi validada e nem comparada (qSOFA vs. SOFA). Dentro de programas de melhoria de qualidade e estratégia de segurança de pacientes, identifica pacientes com alto risco de deteriorização clínica deve ser prioritario ao reconhecimento do risco de obito.

A presença de apenas um critério do SOFA, como hipotensão sem uso de drogas vasoativas ou Escala de Coma de Glasgow 13 ou 14, pode significar ausência de disfunção orgânica. Pacientes com hiperlactatemia isoladamente, sem outra disfunção documentada, ambém seriam considerados como portadores de in ecção sem disfunção, o que gera discussão sobre método proposto.

O do qSOFA também pode ter queda de especificidade para doenças cardíacas, respiratórias ou neurológicas, já que envolvem diretamente uma ou mais variáveis propostas.

Conceitualmente, pacientes sem hipotensão e com hiperlactatemia apresentam maior risco de morte, sem considerar deteriorização clínica. ${ }^{\circ}$ O impacto da hiperlactatemia vem sendo atualmente mais valorizado eforçado, como fator de risco independente de óbito, quando acima de $4 \mathrm{mmol} / \mathrm{L}$ e entre $\circ 7^{\circ}$ e $\circ 28^{\circ}$ dia após a ressuscitação e manejos adequados das dire trizes do SSC. ${ }^{13}$

Abaixo, na tabela 3 , resumidamente, são descritas as principais mudanças entre as definições do Sepsis-2 e Sepsis-3.

Tabela 3. Diferenças conceituais para o estado da 的O ${ }_{2}$, Pressão Arterial Parcial de Gás Carbônico; RNI, Razẫo Normalizada Internacional; PAS, Pressão Arterial Sistólica; PAM, Pressão Arterial Média; $\mathrm{SaO}_{2}$, Saturação Arterial Parcial de Oxigênio)

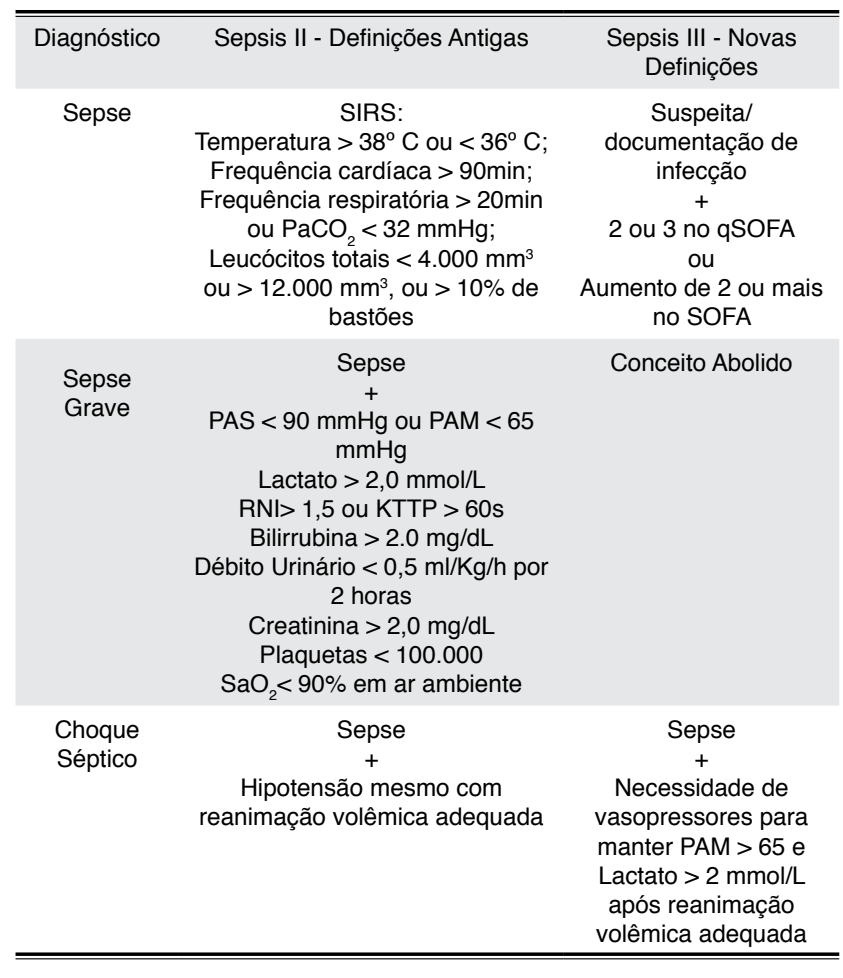


SRIS E SEU PAPEL USUAL DE RECONHEClENTO DA SEPSE

Por anos, os critérios de SRIS foram comumente utilizados para o diagnóstico e o reconhecimento da infecção, sendo, portanto, considerados integrantes das definições anteriores e fisiopatológicas da sepse. A presença de dois critérios de SRIS já caracterizaria o estado de sepse.

Com os avanços de estudos sobre a fisiopatologia da sepse, observou-se que a necessidade de dois ou mais critérios poderia significar uma queda da especificidade para identificação da infecção, principalmente após as primeiras 24 horas de admissão em ambiente crítico e em pacientes idosos, onde a temperatura a frequência cardiaca e respiratória podem estar alteradas por outras razoes, como insuficiência cardíaca ou tromboembolismo pulmonar. "Nestes casos, as criticas sobre a especificidade dos critérios de SRIS se baseiam no fato de que cerca de $90 \%$ dos pacientes em ambiente critico e 50\% dos pacientes em unidades abertas apresentam dois ou mais critérios durante sua

Apesar disto, sabidamente, existe uma relação inversamente proporcional entre o intervalo de tempo entre a evolução de um estado inflamatorio inespe cífico para sepse e o número de critérios de SRIS: prevalência de infecção e bacteremia aumenta com o aumento do número dos critérios de SRIS e, consequentemente, significando estados mais graves e com maior mortalidade.

Porém, em estudo recentemente publicado, constatou-se que os pacientes de UTI com sepse presumida com infecção, disfunção orgânica e risco de mortalidade significativo poderiam nâo preencher os critérios de SRIS em $12 \%$ dos casos. Ou seja, dos pacientes admitidos na UTI, nas primeiras 24 horas de internação, os critérios de SRIS excluem um paciente a cada $8 \mathrm{com}$ diagnóstico de infecção e disfunção orgânica. ${ }^{4}$

As tentativas de simplificação dos critérios de SRIS também não foram conclusivas. Em uma análise realizada ajustando o uso de dois criterios para definição de sepse comparativamente a quatro critérios, houve um crescimento importante da mortalidade a cada elemento adicional até o quarto critério. Isto, por sua vez, também contribui para a redução de sua especificidade.

Apesar das criticas aos componentes dos criterios de SRIS, a presença de infecção e de dois critérios ja está associada a uma mortalidade intra-hospitalar de $5 \%$ a $16 \% .^{16,17}$

As possíveis mudanças ou desencorajamento do uso de criterios diagnosticos e screening bem co- nhecidos podem gerar uma maior espera e falhas em protocolos já estabelecidos para uma condição que exige uma intervenção rapida e eficaz para se sucesso, principalmente em hospitais que não são Emos acadêmicos. ${ }^{12}$

Em discussão recente entre especialistas e não especialistas em medicina crítica nos Estados Unidos, o uso do SOFA sobre os criterios de SRIS e pouco comum, principalmente em hospitais menores, apesar do seu reconhecimento como ferramenta prognostica. Para seu uso ser efetivamente instituido e seguindo as recomendações da Campanha de Sobrevivência à actrace e Tal pros novas ferramentas em diretrizes vigentes. Tériosesso educacional já estabelecido, com os cri-

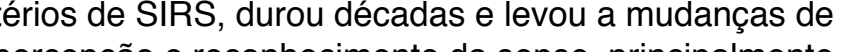
percepcao e reconhecimento da sepse, principalmente cionsto Consequentemente, o reconhecimento extensivo dos chlerios de SRIS identifica precocemente os pacines emse, o que resulta numa rápida ressuscitação inicial e inserção de condutas nas diretrizes
propostas para melhora de desfechos e redução da mortalidade. ${ }^{5,17}$

\section{USO DE ESCORES PREDITORES E MARCADO-} RES DE DESFECHOS ADVERSOS DA SEPSE NO

O grande desafio do tratamento da sepse é a efetividade do bundle, mantendo-se coerência e metas bem estabelecidas, conforme os últimos consensos. E, para tanto, as validaçoes de escores e marcadores de deteriorização clínica devem ser prioritários no ambiente de UTI.

A chegada no hospital, no pronto-atendimento, 0 qSOFA tem sido defendido como ferramenta de triagem eficaz. Em uma coorte multicentrica, realizad especificamente em prontos-socorros de centros europeus, envolvendo cerca de 900 pacientes, o qSOFA o SOFA tiveram boa performance para predizer mortalidade intra-hospltalar, tendo uma sensibilidade de $70 \%$ e especificidade de $75 \%$, enquanto os criterios de SRIS tiveram elevada sensibilidade (93\%), porem especificidade inferior $(27 \%)$. Esta superioridade se manteve na análise dos desfechos secundários, como risco de admissão na UTI e a combinação entre a admissão e a permanência superior a 72 horas. ${ }^{18}$

A hiperlactatemia também foi analisada, num modeo secundário ajustado, para prever mortalidade intra- -hospitalar. Esta, quando associada ao qSOFA $\geq 2 \mathrm{e}$ comparada às definições anteriores de sepse grave, pelo SRIS, não demonstrou superioridade, com áreas abaixo da curva similares: 0,80 (95\% IC, $0,75-0,85$ ) vs. $0,80(95 \% \mathrm{IC}, 0,74-0,85)$. $1.8 \mathrm{C}$

Tanto os criterios de SRIS quanto o SOFA vêm sendo estudados e comparados, em acurácia, às outras ferramentas de estratificação de risco, como o MEWS (Modified Early Warning Score) e o NEWS (National Early Warning Score). Este ultimo corresponde ao MEWS modificado no Reino Unido.

Em uma coorte recente, envolvendo pouco mais de 30.000 pacientes, o qSOFA foi mais acurado em predizer mortalidade hospitalar e encaminhamento do paciente para UTI, seja do pronto-socorro ou unidade aberta, por piora clinica, do que o SRIS. Porém, ambos foram menos acurados do que os escores de alerta precoce (MEWS e NEWS). ${ }^{19}$

Dos pacientes que tiveram deteriorização clínica, 0 reconhecimento da piora, com um aumento acima de 2 critérios de SRIS, ocorreu somente cerca de 17 horas depois da suspeição da infecção, enquanto aqueles com qSOFA $\geq 2$ foram de apenas 5 horas. ${ }^{19}$ Os escores de alerta precoce, apesar de serem mais acurados, podem lentificar a identificação destes pacientes pela complexidade de obtenção de valores e pela necessidade de calculadoras programadas com treinamento de equipe multidisciplinar. ${ }^{20}$

No ambiente crítico, a reprodução dos resultados no departamento de emergência tem sido estudada. Em uma coorte observacional realizada em centros australianos envolvendo mais de 180.000 admissões, relacionou-se a capacidade prognóstica do SOFA qSOFA e SRIS em predizer mortalidade ou aumento do tempo de permanência na UTI nas primeiras 24 horas de internamento. O SOFA demonstrou-se muito superior na capacidade discriminatória às outras ferramentas, havendo, inclusive, forte superioridade quando comparada diretamente com O qSOFA, com incremento estatistico de 0.146 (99\% IC, 0.142-0.151).

Reiterando resultados encontrados em estudos semelhantes, o SOFA corresponde a melhor ferramenta para predizer mortalidade e internamento prolongado em ambiente crítico..$^{21,22}$

CONSIDERAÇÕES FINAIS

As novas definições de sepse, que consideram o estado de infecção, devem ser reavaliadas. Não se tem um completo dominio sobre o papel de fatores imunes e não-imunes na resposta às alterações orgâ- nicas e não se sabe até que ponto o estado inflamatóro corresponde a uma resposta adaptativa presumida. $O$ reconhecimento do qSOFA como escore auxilia para identificação de pacientes sépticos deve ser encorajado apesar dos desconhecimento usual das equipes assistenciais dos hospitais em nossa realidade. A necessidade de inclusão do método nos protocolos instifucionais pode ser útil e em carater complementar aos criterios de SRIS. Um processo educacional e de informaçáo persistente pode tornar o uso destes escores mais comum e diminuir os erros de interpretação Aschimento sistemático.

A superioridade dos escores mais comuns não está bem-definida, principalmente entre o qSOFA e os criterios de SRIS. O modelo estatístico desenvolvido nos estudos recentes visam predizer morbimortalidade $e$ não são validados primariamente como estrategias de triagem de pacientes. Além disto, as comparações são ñ̃o são ferramentas padrão-ouro para o rastreio da sepse, pela faltantas padrão-ouro para o rastreio da específicos.

Carecem, ainda, estudos para análise prospectiva e que sejam desenhados visando a validação das ferramentas para screening com uma estrategia de treinamento das equipes assistenciais, a fim de integração com os programas de qualidade e segurança do paciente.

O conhecimento consolidado dos critérios de SRIS garante a familiaridade da equipe multidisciplinar para ações urgentes diante da sepse.

No ambiente crítico, é imperioso o investimento em desenvolvimento de métodos capazes de prever desfechos que não são comumente analisados, como readmissão durante qualquer período do internamento hospitalar ou risco de retorno precoce, nas primeiras 24 horas da alta para unidade aberta.

Adicionalmente, carecem estudos com abrangencia e inclusão de pacientes com longo tempo de permanencia em ambiente hospitalar, ou portadores de infecções multirresistentes nosocomiais, que são comuns em unidades criticas de paises em desenvolvimento. Isto exige um seguimento mais prolongado e análises de tendência de outras mensurações dos escores durante o internamento.

\section{REFERÊNCIAS}

1. Funk, DJ; Parrillo, JE; Kumar, A. Sepsis and Sep2. Silva E, Pedro MA, Sogayar AC, Mohovic T, Siva $C L$, Janiszewski M, Cal RG, de Sousa EF, Abe TP, 
de Andrade J, de Matos JD, Rezende E, Assunção M, Avezum A, Rocha PC, de Matos GF, Bento AM, Corrêa $A D$, Vieira PC, Knobel E; Brazilian Sepsis Epidemiological Study. Brazilian Sepsis Epidemiological Study (BASES study). Crit Care. 2004;8(4):R251-60.

3. Members of the American College of Chest Physicians/Society of Critical Care Medicine Consensus Conference Committee (1992) Definitions for sepsis and organ failure and guidelines for the use of innovative therapies in sepsis. Crit Care Med 20:864-874.

4.Dellinger RP,Levy MM,Rhodes A, et al. Surviving Sepsis Campaign: international guidelines for management of severe sepsis and septic shock: 2012. Crit Care Med 2013;41:580-637.

5. Cohen, J, Vincent, J-L, Adhikari, NKJ et al. Sepsis: a roadmap for future research. Lancet Infect Dis. 2015;15. 581-614.

6. Singer $M$, Deutschman CS, Seymour CW, Shankar-Hari M, Annane D, Bauer M, et al. The Third International Consensus Definitions for Sepsis and Septic Shock (Sepsis-3). JAMA. 2016;315(8):801-10.

7. Shankar-Hari M, Phillips G, Levy ML, et al. Assessment of definition and clinical criteria for septic shock.JAMA.doi:10.1001/jama.2016.

8. Balk RA . Systemic inflammatory response syndrome (SIRS) Where did it come from and is it still relevant today? Virulence, 2014. 5:20-26.

9.Casserly B, Phillips GS, Schorr C, et al. Lactate measurements in sepsis-induced tissue hypoperfusion: results from the Surviving Sepsis Campaign database. Crit Care Med. 2015;43(3):567- 573.

10. Seymour CW, Liu V, Iwashyna TJ, et al. Assessment of clinical criteria for sepsis.JAMA. doi: 10.1001/ jama.2016.

11. Rhodes A, Evans L, Alhazzani W, et al.Surviving Sepsis Campaign: International Guidelines for Management of Sepsis and Septic Shock. Intensive Care Med, 2016.

12. Vincent JL, Martin GS, Levy MM.qSOFA does not replace SIRS in the definition of sepsis. Crit Care. 2016 Jul 17;20(1):210. doi: 10.1186/s13054-0161389-z.

13. Song JE, Kim MH, Jeong WY, Jung IY, Oh DH, Kim YC, Kim EJ, Jeong SJ, Ku NS, Kim JM, Choi JY. Mortality risk factors for patients with septic shock after implementation of the surviving sepsis campaign bundles. Infect Chemother 2016;48:199-208.

14. Kaukonen KM, Bailey M, Pilcher D, Cooper J. Systemic Inflammatory Response Syndrome Criteria in Defining Severe Sepsis. N Engl J Med 372;2015.

15. Liao MM, Lezotte D, Lowenstein SR et. Al. Sen- sitivity os systemic inflammatory response syndrome for critical illness among ED patients. Am J Emerg Med 2014: 32:1314-25

16. Brun-Buisson $C$. The epidemiology os the systemic inflammatorry response. Intensive Care Med 2000, 26 suppl. 1 .

17. Sprung, CL, Schein RMH. To SIRS with love-An open letter. Crit care Med;2016.

18. Freund, $Y$, Lemachatti N, Krastinova E et Al. Prognostic Accuracy os Sepsis-3 Criteria for In-Hospital Mortality Among Patients With Suspected Infection Presenting of the Emergency Department. JAMA; 2017:301-308.

19. Churpek MM, Snyder A, Han X, Sokol S, Pettit N. qSOFA, SIRS, and early warning scores for detecting clinical deterioration in infected patients outside the ICU. AJRCCM Articles in press. 2016.

20. Santucci, MC, Cavalcanti AB. Chegando a um consenso: vantagens e desvantagens do Sepsis 3, considerando países de recursos limitados. Rev. Bras. Ter Intensiva, 2016 (4):361-365.

21. Lamontagne, F, Harrison DA, Rowan KM. qSOFA for Identifying Sepsis Among Patients With Infection. JAMA, Vol. 317 n3, 2017.

22. De Backer D, Dorman T. A Continuous Move Toward Better Care of Patients With Sepsis. JAMA, Viewpoint Jan 2017.doi:10.1001/jama.2017.0059.

1- Serviço de Terapia Intensiva do Hospital Santa Izabel

Endereço para Correspondência:

trindaderaphaell@gmail.com 